\title{
MODELLING THE STATE OF SOCIAL-ECONOMIC ENVIRONMENT UNDER THE CONDITIONS OF UNCERTAINTY
}

\author{
Poletaikin A.N., Associate Professor \\ Siberian State University of Telecommunications and Information Science, \\ Novosibirsk, Russia \\ E-mail: alex.poletaykin@gmail.com
}

\begin{abstract}
The article covers the hybrid approach to the modeling of the state of social-economic environment under the conditions of uncertainty based on the fuzzy simulation-optimization model with the use of compromise mechanism by the determination of the consumer's satisfaction level. The process of goods promotion to the market has been chosen as the social-economic process of interest; it is a multidimensional process, tightly connected with the registration and processing of social and economic information that represents the set of phenomena, events and facts taking place in the social-economic environment of the market. While conducting the research the hypothesis has been developed and confirmed that the uncertainty and inconsistency of the initial data can be extinguished with the help of the apparatus of fuzzy logic by forming the fuzzy knowledge base of the intelligent system of decision making support and the use of the knowledge for forming the meanings of the main parameters of the goods realization process used in optimal decision making by the goods promotion to the consumer. The proposed hybrid model based on the hard formalized initial data develops the forecast of goods realization to the consumer that is optimal according to the social and economic indexes. The efficiency of the use of the developed model is confirmed by the research of its functioning efficiency in the structure of the system of decision making support.
\end{abstract}

\section{KEY WORDS}

Good promotion to the market, goods realization, social-economic environment, simulationoptimization approach, fuzzy modeling, fuzzy knowledge base, hybrid model, functioning efficiency, compromise decisions, system of decision making support.

The modern market of consumer goods is a complicated, not distinctly definite socialeconomic system, whose thorough analysis is necessary for good management. By the way, the special attention is paid to the state and behavior of a final consumer at the market. They are determined by a large number of various social, economic and psychological factors and sometimes go against pure logic [1]. Considering the economic behavior, one may mention, that as any behavior it has to be aimed at the result and characterized by such a definition as "efficiency». In the most general aspect, it is a behavior connected with the enumeration of economic alternatives with the purpose of reasonable choice, i.e. the choice where the expenses are minimized and net profit is maximized. The background for the economic behavior is the economic consciousness, economic thinking, economic interests, and social stereotypes. By this each phenomenon carries its weight and forms one or another type of economic behavior in its way [2]. From the point of view of sociology and the sociological theory of interaction being formed nowadays, the main emphasis is on the study of social laws and ways of the behavior of mass consumer. The sociologists are trying to answer essentially the question about the reasons and forms of social contradictions: what conformities pursue the social activities, what dominates - enforcement or compromise. Indeed the dilemma «conflict-consensus» or «tensionstability» is saved as the most important scientific problem of all theoretical sociology of the last hundred years [3]. The economics of compromise behavior is connected with the understanding of compromise role in economics, which is usually interpreted as a concession to some demands, refusal from the part of one's own demands in favor of the agreement with other economic subject. The compromise in economics includes such characteristics as the unification, 
cooperation of the interacting parties and at the same time some characteristics of mutual excerption, confrontation, conflict that appear in the relationship between them. The compromise characterizes the essence of economic conflict regulation to the best advantage as it is targeted at the reduction of its efficiency. In connection with it, it is rather important to create and implement new information technologies, taking into account a high uncertainty and inconsistency of the demonstration of personal characteristics of mass consumer in socialeconomic environment such as the market of consumer goods.

The task of the forecast of the consumer goods realization. Let's consider the task of the forecast of the consumer goods realization. This task is shaped as a multifactor information process which demands the adequate performance and optimization with the help of modern information technologies.

The analysis of the modern approaches to the forecast task solution in market promotion allowed to distinguish the following categories of the initial data:

- time series of the realizations for the previous period, including revenues, expenditures, surplus, profit etc.;

- statistic estimation of the demand at the regional market;

- source documents, showing the essence of subject field (invoices, bills of lading, account books, history of realizations etc.);

- expert estimations of the characteristics of goods realization process, such as: formal ways of the performance of the main indicators of goods realization under the conditions of uncertainty; the principles of goods classification and coding; formal-logic rules of market promotion of goods; the peculiarities of the proposed task solutions of goods promotion to the consumer etc.

These categories make up two groups of initial data: 1) quantitative ones, got from the available data that the object has with the help of automated processing and 2) qualitative ones that make up «crystallized» information about the subject field in the form of documentation and experts' competence. For the building of the model of the subject field, it is very important to understand the nature of exactly qualitative data. With the use of the given in [4] analysis methodology of the properties of qualitative data, one has succeeded to set up their classified structure, influencing the decision making while market promotion of goods.

The interpretation of the data essentially depends on the order of their appearance in time, that is extremely important by the modeling of cause-effect relationship between them, among those are also distinguished the causal, definite and correlation ones. The data are numerous, though not fully determined - the category of stochastic data is distinguished, it is about the influence on the goods demand of such factors as seasonality, weather conditions, social temperature etc. - and they are insufficient because of the number of end consumers. All the data, required for the use in the model, are incomplete and insufficiently agreed; they are subject to the constant renewal and agreement. Therefore that for the structuring and formalization of such data, it is reasonable to use the apparatus of artificial intelligence for the work with the knowledge, forming the base of knowledge, containing the well-structured information about the subject field, for the intellectual system of the support of decision making (ISSDM); the system, which substrate is made up by the hybrid model of goods promotion (HMGP), generates the compromise decisions on the basis of the above-mentioned data [5].

Nowadays a lot of factors, influencing the realization process, have been studied. The majority of them represent stochastic-determined social-economic indexes, many of those can be systematically described as inaccurate values, because of the proved circumstance that the complicated dynamic process is adequately described with the help of fuzzy logic that is a constant one by definition [6]. By this the influence of the factors onto the system is determined by the estimations having been got on the basis of the model of fuzzy logic, taking into account the grading according to the classification of goods, with the attraction of a group of experts as the main source. Due to such task setting, let us further call the considered factors and indexes of realization as fuzzy factors (FF) and indexes (FI), quantitative characters of goods classification, regarding those we get the estimations of FF and $\mathrm{FI}$, let us call them feature characters (FC) [7]. By such initial data for the solution of the given task, it is necessary, first of all, to fulfill the sub-tasks of the forming of knowledge base: 
1. To form the number of FF, FI and main FC, and to realize the problem classification of goods as well.

2. To carry out the multi aspect classification of goods according to the variety of characters, because not all the factors can be adequately considered regarding the qualitative FC.

3. For all FF and FI to introduce the linguistic variables and determine: term-set of their linguistic meanings; the membership functions of the meanings on some universal sets of meanings; the algorithm of the influence of FF estimation onto the probabilistic estimations of the process of goods promotion to the consumer.

The result of the task solution of goods promotion to the consumer represents itself the sales forecast - the document of tabular format, containing the forecast data about the quantity of concrete units of goods that can be realized under the current conditions for the relevant period. The optimal variant of such forecast as shown in [8,9], because of the infinity of the field of admissible solutions and a great number of parameters, influencing this solution, can be made up combinatorially with the help of the apparatus of genetic algorithms. In this case the task of multi criteria optimization by decision making takes place.

The methodology of the modeling of the state of social-economic environment. In order to understand the essence of the assigned task, let us consider the methods and ways of the formalization of the initial data. It was above-mentioned that the initial information, necessary for the solution of the assigned task, represents itself the estimation of the qualitative factors for the realization of goods in the region. The collection, systematization and research of these data are carried out based on the object of informatization as a rule. The getting of the initial data according to the factors of realization has been carried out with the use of individual communicative methods of knowledge acquisition about the subject field, for their structuring in [10] it is proposed to use the generalized object-structured approach and built on it the algorithm of object-structured analysis (OSA) of subject field, allowing to optimize and regulate the procedures of knowledge structuring. The main postulates of this paradigm are borrowed from the object-oriented projecting and expanded, in particular, the modularity is a segmentation of task into the subtasks and "possible worlds". It allows to build the global databases with the opportunity to distinguish the local tasks with the help of horizontal and vertical cuts into the separate modules of space - the description of subject field. OSA means the disaggregation of subject field into the eight strata or layers (vertical analysis), as a rule, and the forming of the matrix of OSA $\left\|E_{m n}\right\|$, where $m$ - a number of level, $n$ - a number of strata and $E$ belongs to the set of all concepts of subject field. The structure of $E$ matrix is shown in Table 1. Then the disaggregation is fulfilled according to the levels from the problem to the subtask (horizontal analysis). If it is necessary the number of strata may be increased.

Table 1 - Matrix of object-structured analysis

\begin{tabular}{|c|c|c|c|c|c|c|}
\hline 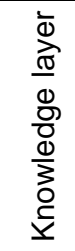 & Knowledge type & Strata & $\frac{\bar{d}}{\frac{\bar{d}}{\overline{0}}}$ & 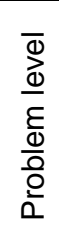 & 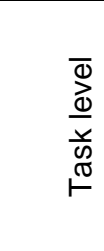 & $\begin{array}{l}\bar{d} \\
\frac{d}{0} \\
\frac{k}{0} \\
\frac{\pi}{0} \\
\frac{0}{0} \\
\omega\end{array}$ \\
\hline S_1 & WHAT FOR & Strategic analysis & $e_{11}$ & $e_{12}$ & $e_{13}$ & $e_{14}$ \\
\hline S_2 & WHO & Organizational analysis & $e_{21}$ & & \multirow{7}{*}{$\begin{array}{c}\text { Set of a } \\
\text { concept } \\
\text { of subje } \\
\text { field }\end{array}$} & \\
\hline S_3 & WHAT & Conceptual analysis & $e_{31}$ & & & \\
\hline S 4 & $\mathrm{HOW}$ & Functional analysis & $e_{41}$ & & & \\
\hline S_5 & WHERE & Spatial analysis & $e_{51}$ & & & \\
\hline $\mathrm{S} 6$ & WHEN & Temporary analysis & $e_{61}$ & & & \\
\hline S_7 & WHY & Causal analysis & $e_{71}$ & & & \\
\hline $\mathrm{S} 8$ & HOW MANY & Economic analysis & $e_{81}$ & & & \\
\hline
\end{tabular}


In its turn the knowledge of each strata undergo the further analysis and are divided into the constituents $\left\|e_{i j}\right\|$. The matrix $E$ is a dissymmetric one, as the part of $E$ elements can be subject to the decomposition and another part represents the basic atomic concepts from $K$. For the detailed practical structuring of knowledge of subject field, it is reasonable to use the algorithm of OSA, based on $E_{m n}$ matrix filling.

In the result of the textual and object-structured analysis and expert estimation as well, three categories of indicators, characterizing the process of goods promotion to the consumer, have been determined [11]:

- Category 1 indexes that may be adequately described with the use of the theory of chance and mathematical statistics, and as a result, they are modeled by the statistical methods (e.g. the frequency of goods demand, availability of money by the consumer etc.);

- Category 2 indexes that, because of the some circumstances (high uncertainty, difficulties of experts in the determination of description laws), cannot be adequately described neither statistically nor by the laws of the theory of chance (e.g. weather conditions);

- Category 3. indexes that fundamentally cannot be estimated quantitatively with the help of any mathematical methods (e.g. destination).

Taking into account such a variety of initial data, the majority of which are characterized by high uncertainty, it has been an attempt of their simulation modeling based on the statistical data, having been got by the way of the long-term observation for the dynamics of these data. However, as the research of the adequacy of the given model has shown, the proposed hypotheses towards the stochastic nature of the initial data turned out to be the untenable ones and didn't give the adequate results of the modeling of the sought output characteristics. As a result, the developed simulation-optimization model of the management of the process of goods realization has shown a low adequacy. In connection with it, the hypothesis has been proposed that the uncertainty and inconsistency of input parameters can be liquidated by the apparatus of fuzzy logic by the way of the forming of indistinct knowledge base ISSDM and the use of the knowledge for the forming of the meanings of main parameters of the process of goods realization, used by the making of optimal solutions by the goods promotion to the consumer. Such task setting determines the structured scheme of data conversion (Fig. 1) by the getting of the resulting forecast.

Modeling of the state of social-economic environment under the conditions of uncertainty. Let us consider more detailed the initial data related to the category of the qualitative ones, that's why being a subject to the expert estimation.

The first category of indexes can be divided into 2 groups: the data about the good and the data about the demand for the good in the region as well. These indexes are given correspondently in Tables 2 and 4 including the methodological strata (See Table 1) of the given knowledge and quantitative form of its expression (the characteristics of food products are marked with star keys). Each index in Tables 2 and 3 can be also stratified according to the strata "Why». This type of knowledge can be used in the subsystem of ISSDM explanations.

In Table 3 for the indexes of goods realization, showing the essence of human factor, the denomination FF (fuzzy factor) is given in the column "Strata», that is also represented by the membership function. The indexes are grouped according to the influence made as the general ones (for all the goods, of the whole region) and private ones (for a concrete good unit). The data, general for the region, have been got based on the statistical processing of the data of consumers' questionnaires and also the regional statistics management due to each category of initial data.

The second category of indexes is a set of the essential factors and characteristics of traditional (problem) and qualitative classification of goods. A fuzzy model is introduced for each factor. The model is characterized by the membership function $\mu(\omega)$, that may be given in tables or diagrams by the program means, realized in the subsystem of conclusion. 


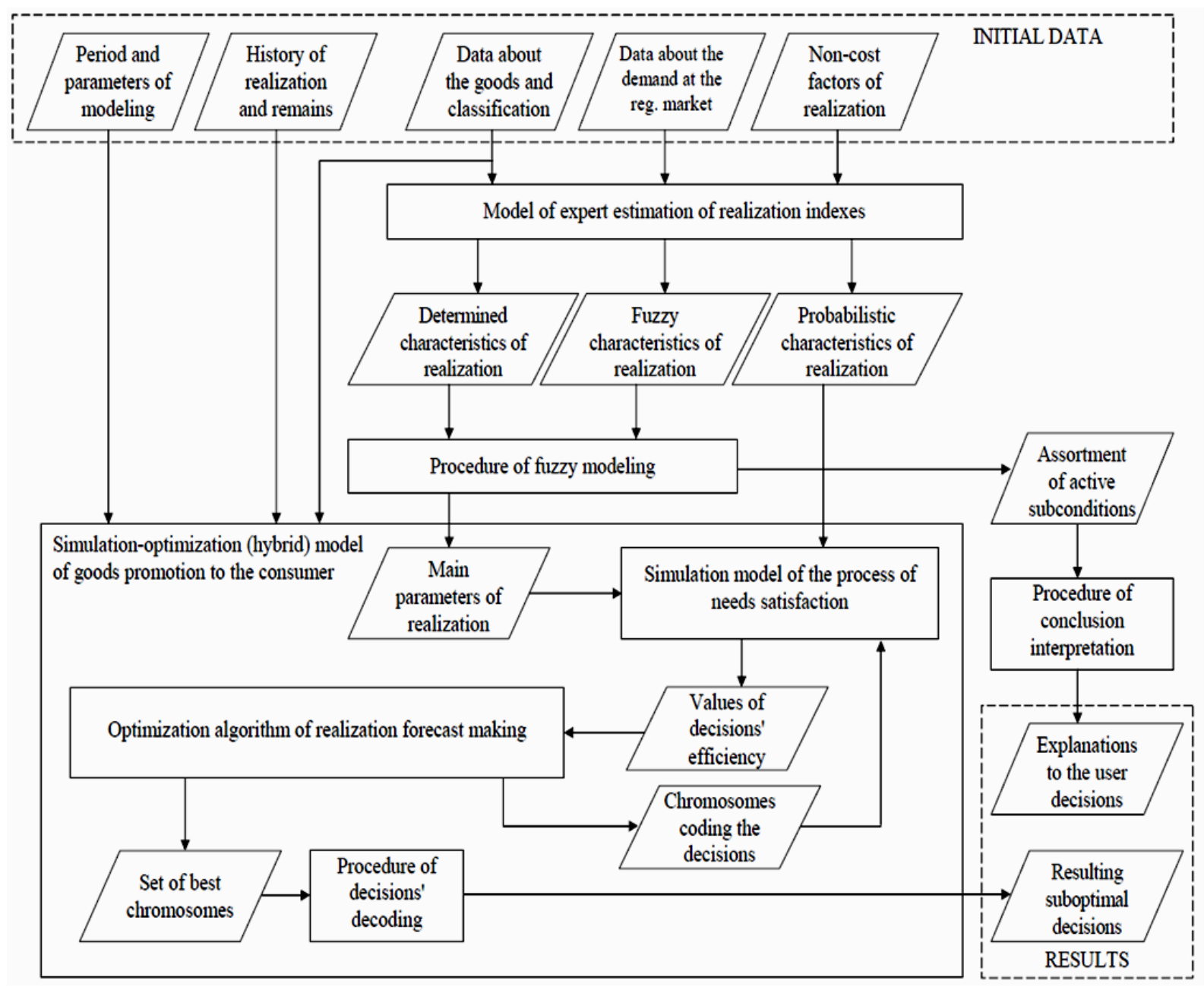

Figure 1 - Structured scheme of data conversion in the process of ISSDM decision making

Table 2 - Possible set of input data about the modeled good

\begin{tabular}{|l|l|l|}
\hline \multicolumn{1}{|c|}{ Input value } & \multicolumn{1}{c|}{ Strata } & \multicolumn{1}{c|}{ Form of mathematical expression } \\
\hline The code of good & What/Where & The code of classificator \\
\hline Realized quantity & How many & Absolutely (pcs.) or relatively (\%) \\
\hline The number of failures & How many & Absolutely (pcs.) or relatively (\%) \\
\hline Frequency of demand & How many & Numbers on the time scale \\
\hline Validity period & How many & Numbers on the time scale \\
\hline Quality & How many & Percentage (probabilistic) estimations \\
\hline Technical characteristics & How many & Percentage (probabilistic) estimations \\
\hline Design & How many & Percentage (probabilistic) estimations \\
\hline Degree of usefulness & How many & Percentage (probabilistic) estimations \\
\hline Way of manufacture & How & The code of classificator \\
\hline Remains of goods at the warehouse & How many & Goods units, percentage \\
\hline Forecast period & When & Time scale \\
\hline
\end{tabular}

Table 3 - Input data about the demand for the good

\begin{tabular}{|l|l|l|}
\hline Input value (FF) & Strata & Form of quantitative expression \\
\hline GENERAL (for the region) & How many & Probabilities \\
\hline Availability of money b the consumer & How many & Statistical estimations, probabilities \\
\hline Cash revenues in the region & How many & Statistical estimations, probabilities \\
\hline Population & How many & Statistical estimations, probabilities \\
\hline Number of family members & How many & Statistical estimations, probabilities \\
\hline Age and gender composition & FF & The membership function \\
\hline Health & FF & The membership function \\
\hline Unemployment & FF & The membership function \\
\hline Living facilities &
\end{tabular}




\begin{tabular}{|l|l|l|}
\hline \multicolumn{3}{|c|}{ Continue of Table 3 } \\
\hline Culture and extra occupational life & FF & The membership function \\
\hline Peculiarities of consumer employment & FF & Probabilities, probabilistic distributions \\
\hline Bandwagon effect & FF & Probabilities \\
\hline Periodicity & When & Probabilistic distributions \\
\hline PRIVATE (for the concrete good unit) & How many & Statistical averages, probabilities \\
\hline The degree of market saturation & How many & Proportions, probabilities \\
\hline Availability of substitution goods & How many & Proportions, probabilities \\
\hline Availability of complementary goods & How many & Proportions, probabilities \\
\hline Availability of abundant analogs for the scarce goods & FF & The membership function \\
\hline Likings and habits of consumer & FF & The membership function \\
\hline Fashion &
\end{tabular}

Fuzzy modeling of the state of social-economic environment. The third category of indexes is officially represented by the total set of their semantic meanings, which are also used in the procedure of fuzzy modeling of the parameters of the process of goods realization. First of all, they are the most important characteristics of the process such as purchasing power and the motivation for the consumer's activity, the probabilities of the realization of the definite group of goods or a concrete good unit as well. Because of the apparent uncertainty of the initial data and impossibility of its settlement by the probabilistic methods, the sub-model has been separated from the structure of simulation model. The sub-model determines the above-mentioned characteristics of the process of goods realization based on the modeling of the initial data with the use of indistinct logic and mechanism of indistinct output. The sub-model has been taken as a basis for the subsystem of conclusion and provides the simulation-optimization model of goods promotion to the consumer by the true data (Fig. 2).

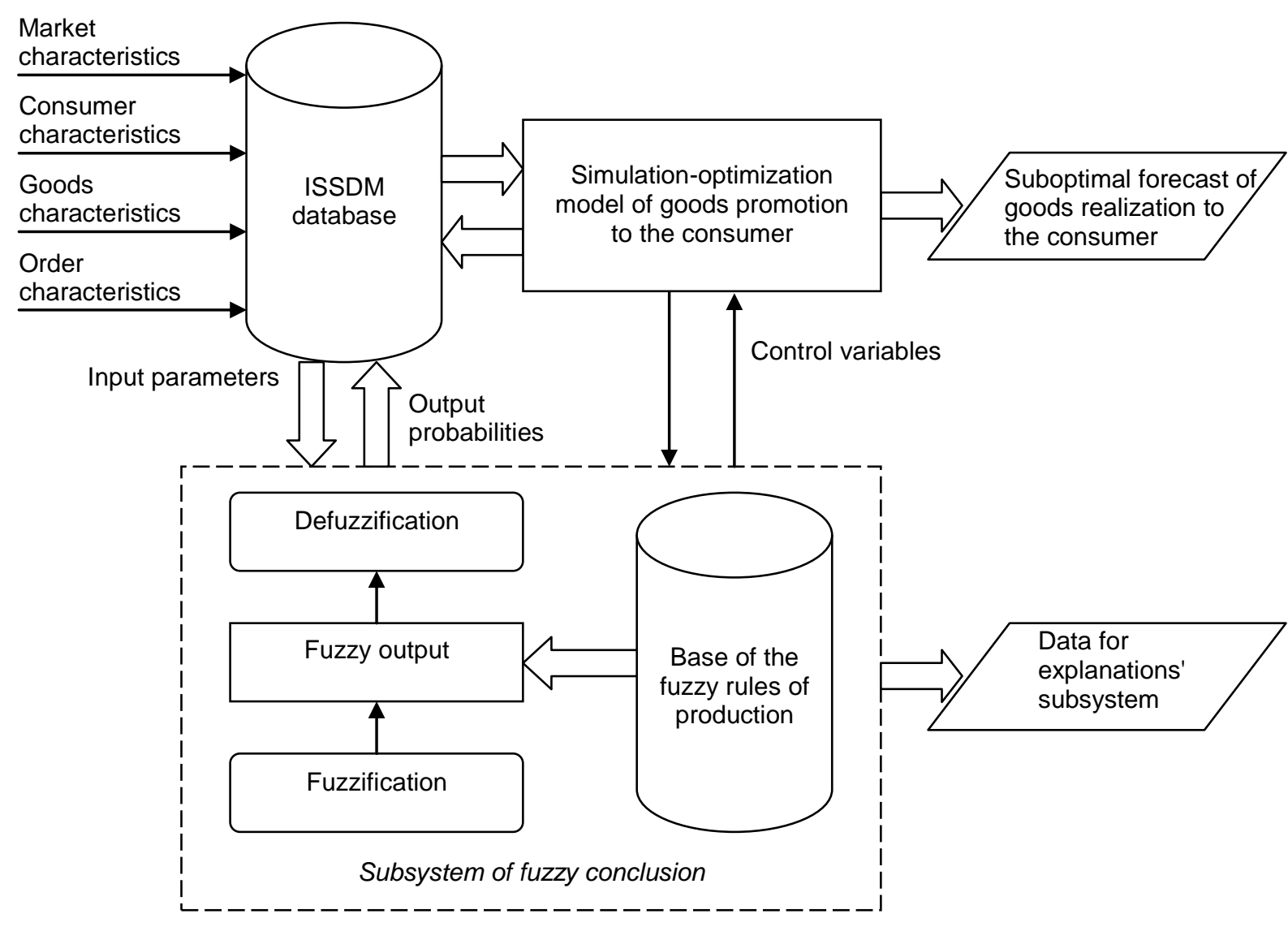

Figure 2 - Structure of the process of fuzzy modeling of realization parameters 
Here the factual meanings of input values go to the database of ISSDM through the interface of the user where they are supported in the actual meanings during one iteration of cycle of goods promotion to the consumer. At each iteration the actual meanings from the database are given at the input of the subsystem of fuzzy output. Under the influence of control signals from the simulation-optimization model this subsystem fulfills the fuzzy modeling of realization parameters and fuzzy output, whose result is the set of actual meanings of the above-mentioned characteristics of the process of goods realization. By this the results of output are accompanied by the instructions in the language, near to the natural one for the output of ISSDM explanations to the consumer through the subsystem.

The determination logic of the characteristics of the process of goods realization is represented by the experts at the example of the realization of computer technique (CT) and got in the process of knowledge extraction in the form of formal-logic rules, written based on the classification of goods nomenclature according to the groups in the terms of input and output indexes of the process of goods realization to the consumer. Some of such rules, formulated for the group of goods «CDs, DVDs»:

IF the geographic location is near the educational institution AND Age is under 40 AND Assortment is wide IT MEANS that the purchase probability is high;

IF the geographic location is the center of the city AND Age is more than 40AND Assortment isn't wide IT MEANS that the purchase probability is low.

The initial set of rules together with the formalized fuzzy factors and characteristics of goods classification represent the initial data for the building of the base of rules of fuzzy output system allowing to work out the corresponding subsystem of ISSDM. The variables, expressed by the crisp set (consumers' occupation, geographic location of the shop etc.) have been detected by analyzing the rules for the determination of input and output variables of the model. The list of crisp variables $\alpha$ is given in Table 4.

Table 4 - Input crisp variable characteristics of CT purchase

\begin{tabular}{|c|c|c|}
\hline Name & Symbol name & Crisp set \\
\hline Spc & Class of goods & $\left\{s_{1} \ldots s_{27}\right\}$ - characteristics of classification of computer technique \\
\hline$\alpha_{1}$ & Geographic location & $\begin{array}{l}\left\{<s_{1}, \text { near educational institution }>,<s_{2} \text {, center of the city }>,<s_{3} \text {, residential }\right. \\
\left.\text { area }>,<s_{4} \text {, office area }>\right\}\end{array}$ \\
\hline$\alpha_{2}$ & Destination & $\begin{array}{l}\left\{<s_{1} \text {, for the house }>,<s_{2} \text {, for the office }>,<s_{3}, \text { professional work with graphics }>\text {, }\right. \\
\left.<S_{4}, \text { commercial }>\right\}\end{array}$ \\
\hline$\alpha_{3}$ & Peculiarities of occupation & $\begin{array}{l}\left\{<s_{1}, \text { work in office }>,<s_{2} \text {, availability of home printer }>,<s_{3} \text {, specialist in IT }\right. \\
\left.\text { sphere }>,<s_{4}, P C \text { user }>,<s_{5} \text {, professional photographer }>\right\}\end{array}$ \\
\hline$\alpha_{4}$ & Social group & $\begin{array}{l}\left\{<s_{1} \text {, elite }>,<s_{2} \text {, ordinary citizen }>,<s_{3} \text {, self-employed individual }>,<s_{4} \text {, officer }>\text {, }\right. \\
\left.<s_{5} \text {, engineer }>,<s_{6} \text {, state employee }>\right\}\end{array}$ \\
\hline$\alpha_{5}$ & Design & $\left\{<s_{1}\right.$, white body $>,<s_{2}$, black body $>,<s_{3}$, strict/classic $>,<s_{4}$, original $\left.>\right\}$ \\
\hline$\alpha_{6}$ & Season & $\begin{array}{l}\left\{<s_{1}, \text { spring }>,<s_{2}, \text { summer }>,<S_{3} \text {, autumn }>,<s_{4} \text {, winter }>,<s_{5} \text {, the end of }\right. \\
\left.\text { summer }>,<s_{6} \text {, the end of December }>,<S_{7} \text {, the beginning of spring }>\right\}\end{array}$ \\
\hline$\alpha_{7}$ & Education & $\left\{<s_{1}\right.$, secondary $>,<s_{2}$, specialized secondary $>,<s_{3}$, higher $>,<s_{4}$, degree $\left.>\right\}$ \\
\hline$\alpha_{8}$ & Weather conditions & $\begin{array}{l}\left\{<S_{1}, \text { sunny }>,<S_{2}, \text { wind }>,<S_{3}, \text { rain }>,<S_{4}, \text { snow }>,<S_{5} \text {, hail }>,<S_{5} \text {, fog }>,<S_{6} \text {, ice }\right. \\
\left.\text { slick }>,<S_{7} \text {, after storm }>\right\}\end{array}$ \\
\hline
\end{tabular}

Among the set of the indexes of computer technique's realization process such ones have been detected, which the experts have characterized with some uncertainty. Based on these indexes 30 fuzzy input linguistic variables $\beta$ have been determined (Table 5).

The general abbreviations for the main terms of linguistic variable in the systems of fuzzy output are given as the elements of term-varieties in Tables 5 and 6. Fuzzy output linguistic variables $\omega$ (Table 6) are determined for the forming of probabilistic parameters of the process model of computer technique's realization. The diagram of membership function is given at Fig. 3. The membership function, expressing fuzzy probability is actually given at Fig. 3. The output variables $\omega_{1}, \omega_{2}$ and $\omega_{3}$, expressed by the membership function are 
transformed during the accumulation process into the probabilistic characteristics of the process of goods realization for the use in the simulation-optimization model of goods promotion to the consumer.

Table 5 - Input linguistic data variable characteristics of computer technique sales

\begin{tabular}{|c|c|c|}
\hline Name & Symbolic name & Term-set \\
\hline$\beta_{1}$ & Age of consumer & $\{\mathrm{VL}, \mathrm{L}, \mathrm{BA}, \mathrm{A}, \mathrm{H}\}$ \\
\hline$\beta_{2}$ & Assortment of goods & $\{L, B A, A, A A, H\}$ \\
\hline$\beta_{3}$ & Cost of a concrete goods unit & $\{L, B A, A, A A, H\}$ \\
\hline$\beta_{4}$ & Population & $\{B A, A, H\}$ \\
\hline$\beta_{5}$ & Sales area & $\{L, A, H\}$ \\
\hline$\beta_{6}$ & Qualification of managers & $\{L, A, H\}$ \\
\hline$\beta_{7}$ & Cost of goods regarding the price in the Internet-shop & $\{\mathrm{NB}, \mathrm{NM}, \mathrm{NS}, \mathrm{Z}, \mathrm{PS}, \mathrm{PM}, \mathrm{PB}\}$ \\
\hline$\beta_{8}$ & Quality of good & $\{L, B A, A, A A, H\}$ \\
\hline$\beta_{9}$ & Balance productivity/price & $\{L, B A, A, A A, H\}$ \\
\hline$\beta_{10}$ & Blown model & $\{L, A, H\}$ \\
\hline$\beta_{11}$ & Innovativeness of good & $\{L, A, H\}$ \\
\hline$\beta_{12}$ & Guarantee period of good & $\{L, A, H\}$ \\
\hline$\beta_{13}$ & Market saturation by the analog goods & $\{L, B A, A, A A, H\}$ \\
\hline$\beta_{14}$ & Delivery time to the point of order & $\{\mathrm{VL}, \mathrm{A}, \mathrm{VH}\}$ \\
\hline$\beta_{15}$ & Presence of abundant analogs of deficit goods & $\{L, A, H\}$ \\
\hline$\beta_{16}$ & Money income in the region & $\{L, A, H\}$ \\
\hline$\beta_{17}$ & Effect of exclusivity demonstration & $\{\mathrm{L}, \mathrm{A}, \mathrm{H}\}$ \\
\hline$\beta_{18}$ & Preferences of the certain goods brands & $\{L, A, H\}$ \\
\hline$\beta_{19}$ & Assortment of the competitors & $\{L, B A, A, A A, H\}$ \\
\hline$\beta_{20}$ & Price policy regarding the market & $\{\mathrm{BA}, \mathrm{A}, \mathrm{AA}\}$ \\
\hline$\beta_{21}$ & Average monthly income of the consumer & $\{L, A, H\}$ \\
\hline$\beta_{22}$ & Sufficiency level of the consumer & $\{L, A, H\}$ \\
\hline$\beta_{23}$ & Emotional state of the consumer & $\{L, A, H\}$ \\
\hline$\beta_{24}$ & Frequency of the Internet use & $\{L, B A, A, A A, H\}$ \\
\hline$\beta_{25}$ & Requirements to the productivity & $\{L, B A, A, A A, H\}$ \\
\hline$\beta_{26}$ & Requirements to the compactness & $\{B A, A, A A\}$ \\
\hline$\beta_{27}$ & Requirements to the period of the autonomous work & $\{L, A, H\}$ \\
\hline$\beta_{28}$ & Motivation of consumer's behavior & $\{\mathrm{NB}, \mathrm{NM}, \mathrm{NS}, \mathrm{Z}, \mathrm{PS}, \mathrm{PM}, \mathrm{PB}\}$ \\
\hline$\beta_{29}$ & Law-obedience & $\{L, A, H\}$ \\
\hline$\beta_{30}$ & Weather conditions & $\{\mathrm{VL}, \mathrm{L}, \mathrm{BA}, \mathrm{A}, \mathrm{AA}, \mathrm{H}, \mathrm{VH}\}$ \\
\hline
\end{tabular}

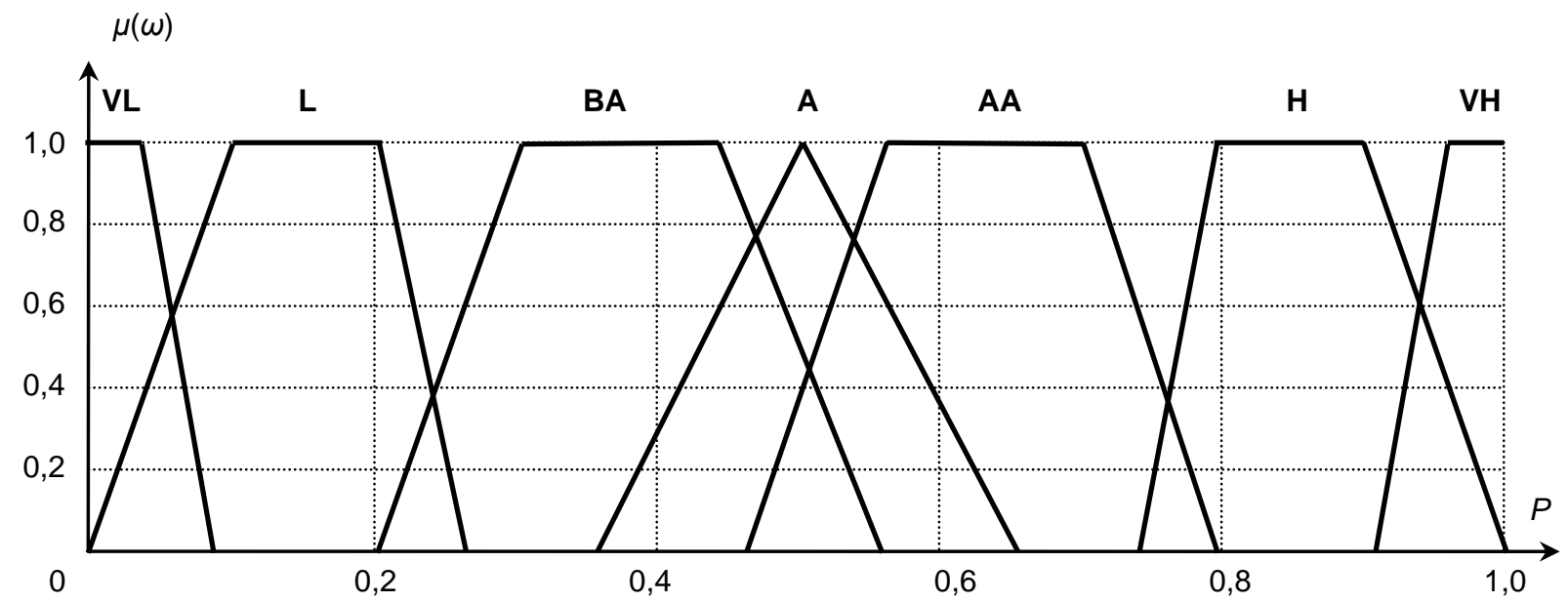

Figure 3 - Graphical representation of the membership function of the output linguistic variables $\omega_{1}$ «The probability of goods realization», $\omega_{2}$ «Purchasing power» and $\omega_{3}$ «The probability of shopping»

Based on the given data, the base of fuzzy productions has been made; fuzzy variables $\beta$ are the parts of antecedents. The crisp variables $\alpha$ and also the problem classification of goods are used for the forming of predicates, being parts of pre-conditions. The rule system is organized in such a way that the maximum correspondence to the 
Mamdani-type inference could take place. It has found the practical use in the tasks of fuzzy modeling nowadays [12]. The example of fuzzy production $R_{1}$ for one of the above-mentioned formal rules and its interpretation according to the semantics of the involved variables is given in Table 7.

Table 6 - Output linguistic variables

\begin{tabular}{|l|l|l|}
\hline Name & \multicolumn{1}{|c|}{ Symbolic name } & \multicolumn{1}{|c|}{ Term-set } \\
\hline$\omega_{1}$ & The probability of the realization of a concrete goods unit & $\{$ VL, L, BA, A, AA, H, VH $\}$ \\
\hline$\omega_{2}$ & Purchasing power (indifferent to the good) & $\{$ VL, L, BA, A, AA, H, VH $\}$ \\
\hline$\omega_{3}$ & The probability of shopping & $\{$ VL, L, BA, A, AA, H, VH $\}$ \\
\hline
\end{tabular}

The antecedents contain fuzzy variables $\beta$ (See table 5 , e.g. the variable $\beta_{1}$ Consumer's age with the term-set $\{V L, L, B A, A, H\}$, whose membership function includes $Z$ shaped $(\mathrm{VL})$, trapezoidal (L, BA), triangular $(\mathrm{A})$ and S-shaped $(\mathrm{H})$ constituents). The crisp variables $\alpha$ (See Table 4) and also the goods classification due to the groups Spc are used for the forming of predicates, being the parts of pre-conditions.

Table 7 - The example of the formal fuzzy output rule

\begin{tabular}{|c|c|c|}
\hline Name & Precondition & Formulation \\
\hline$R_{1}$ & Spc IS $s_{1}$ AND $\alpha_{1}$ IS $s_{1}$ & IF NOT ( $\beta_{1}$ IS A OR $\beta_{1}$ IS H) AND $\beta_{2}$ IS H THEN $\omega_{1}$ IS H \\
\hline
\end{tabular}

The determination of the compromise meaning of the purchasing power of the consumer. The result of the work of fuzzy output subsystem, whose structure is shown at Fig. 2, is the set of three inquired probabilities from the simulation-optimization model. These probabilities have been formed by the way of defuzzification of the accumulated output fuzzy values from Table 6 , one of those $\left(\omega_{2}\right)$ is a compromise representation of the consumer's purchasing power. This index in the achieved crisp form is a basic one in the simulation modeling of the process of goods realization to the consumer. From this point of view the geometric characteristics of the compromise meaning determination of this index is rather illustrative one. This characteristics was developed in 1972 by the outstanding Soviet scientist, academician of Academy of Science USSR N.M. Amosov. Being a medical worker, he paid a great attention to the contradictions of human psyche and modeling of the behavior from the position of compromise both from physiological and psychological points of view. In his fundamental work "Human nature» [13] Amosov developed the model of the determination of human needs, characterized by the certain set of indexes. The model shows the contradictions between human needs and possibilities and allows to determine the compromise characteristics of the interaction with the environment, leading the system «man-environment» to the relative balance. This compromise characteristic of human need is shown at Fig. 4.

The value $P$ - the so-called "plate» (the term of N.M. Amosov) is marked at the absciss axis. It expresses the outside impact, which has to satisfy the need. These plates can look very different - from the video clip registered by the receptor of retina up to the degree of the win in the competition in the comparison of the models «l» and «rival». The zero meaning of "plate» is difficult to be fixed up because of their specifics and different time-scales. The conventional subjective zero is accepted in the diagrams by the given state of adaptation. Parallel to the absciss axis is the horizontal line of "zero feeling" of need's satisfaction, i.e. when the unpleasant feeling has already disappeared but the pleasant one hasn't determined yet. The horizontal line divides the ordinates axis where the dimension of the feeling (value F) is marked, corresponding to the need in the universal scale and expressing the activity of the corresponding nerve center with the components pleasant-up $(\mathrm{Pl}+)$ or upleasant - down (PI-), e.g. hunger - down, saturation - up. 


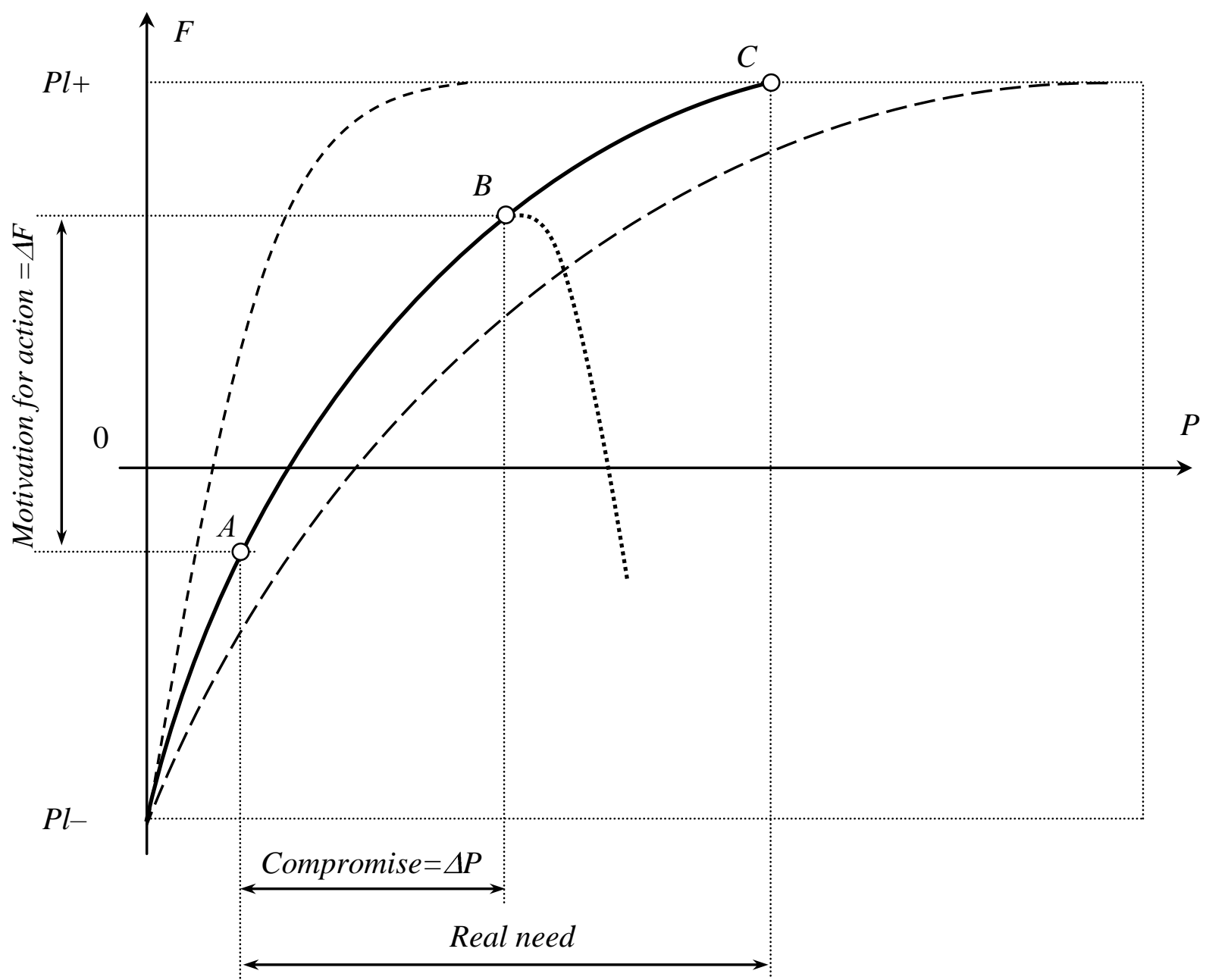

Figure 4 - Geometric meaning of the task of the determination of the motivation for action of the consumer based on the compromise between the needs and possibilities

Due to the degree of pleasantness or unpleasantness, all the feeelings, even quite different in their specifics, are compared. Depending on the correlation optimism-pessimism, the zero line is shifted from the middle up and down, moreover dissimilar for different needs; the significance of different needs is compared according to the minimum and maximum. The main curve is given at Fig. 4. It is a characteristic of the need in the ordinates plate-feeling. At every moment the state of need satisfaction can be marked by the point at this characteristic, e.g. the point $A$ shows that the personality isn't satisfied but not very strongly. With the saturation, the satisfaction increment onto plate unit is decreasing and going to zero. Another place, shown by dots, is possible for some needs, i.e. satisfaction decreasing because of the saturation. The full saturation is marked with the point $C$ at the upper horizontal. The plate corresponding to it is called the maximum need or level of aspiration. It is a very important characteristic for sociological models. The point $C$ is very conventional as the maximum aspirations are not final ones. That's why it is reasonable to use the real aspirations - a certain point $B$, not giving the full satisfaction, but corresponding to some compromise, which is expressed by the increment of plate $\Delta P$ from the point $A$ to the point $B$ - the real need of the individual; and its satisfaction will obligatory give the increment $\Delta F$ - exactly this value represents the actual motivation for action of the consumer.

If the aspirations are satisfied consequently, this level of plate will not give happiness and the satisfaction will decrease. In such a way the adaptation appears; in model expression it means the shift of point $C$ to the right - the increasing of aspiration level (the curve shown as a long dot line at Fig. 4.). On the contrary if the plate is decreasing 
systematically, at first person feels not very satisfied then he gets used to and his level of aspiration is decreasing - point $C$ is shifted to the left (the curve shown as a short dot line at Fig. 4). The limits of compromise adaptation for different needs are also unequal. For instance, it is rather difficult to adapt to hunger, but quite possible to the absence of the Internet. The forming of aspiration level is a very complicated process, but it needs to be modeled, e.g. by making significant some factors [14]. By this the following admissions are possible: 1) initial average state; 2) the reality of achievement; 3) compromise state in the given social group regarding a higher one.

The development of hybrid model of goods promotion to the consumer. Based on the studied characteristics of the process of goods realization one can develop the probabilistic model of typical social process of need satisfaction that being included into the interaction with the optimization algorithm, forms the hybrid simulation-optimization model, shown in the lower part of Fig. 1, and being the main means of support of decision making in ISSDM. The model consists of two interrelated components: 1) statistic simulation model of the system of mass service of CT consumers at the regional market and 2) optimization genetic algorithm developing the suboptimal decisions regarding the promotion of CT to the consumer.

Simulation model describes the demand directly in the process of its satisfaction, in contact with the consumer and in fact being the source of the data and knowledge not only about the actual consumption of goods for the period but also about the character of demand in the region. At each operation cycle the model generates the virtual environment with the given parameters, decoded from the chromosome, whose source is GA (genetic algorithm). Although, based on the fact that it operates with the help of the real data from ISSDM database, which part has been got by the way of fuzzy modeling of the parameters of realization process with the immediate interaction with the real environment, it is more reasonable to call the environment of the given system of mass service as pseudo-virtual one. In the result of one operation cycle of the model, the numerical estimate of integral criterion of efficiency is formed; it is estimated based on the private criteria.

The material for the analysis is the set of the forecasts realization, formed in OSA terms (see Table 1). The forecast realization generally represents the variety of sequences $R_{1}\left\{S_{1}, \ldots, S_{N}\right\} \ldots R_{M}\left\{S_{1}, \ldots, S_{N}\right\}$, its attributes $S_{i}$ show the parameters of realization process. Minimal set of such attributes: $S_{1}$ : Code_good and $S_{2}$ : Quantity_realization. For the determination of quantitative characteristics the definition of large-scale forecast is introduced, that structure is given in Table 8, where the additional parameters are included into the set $\{S\}$. These parameters show space $S_{3}$, time $S_{4}$ and functional $S_{5}$ aspects of realization process.

Table 8 - Structure of the large-scale forecast of good realization

\begin{tabular}{|l|l|l|l|l|l|}
\hline \multicolumn{1}{|c|}{ Parameter } & \multicolumn{1}{c|}{$S_{1}$} & \multicolumn{1}{c|}{$S_{2}$} & \multicolumn{1}{c|}{$S_{3}$} & \multicolumn{1}{c|}{$S_{4}$} & \multicolumn{1}{c|}{$S_{5}$} \\
\hline Knowledge layer & S_3 & S 8 & S_5 & S_6 & S 4 \\
\hline Knowledge type & WHAT & HOW MANY & WHERE & WHEN & HOW \\
\hline \hline Sequence $R_{i}$ & $\{\mathrm{~T}\}$ & Q(T) & $\{\mathrm{C}\}$ & $\{\mathrm{D}\}$ & $\{\mathrm{H}\}$ \\
\hline \hline
\end{tabular}

Parameter $S_{3}$ : Realization channel represents the indicator of operation localization in the modeled process. Parameter $S_{4}$ : The realization date determines the date of operation fulfillment. The functional peculiarity of realization shows the way how the good has been sold: as a stand-alone device or in an item. This aspect of the process is set by the parameter $S_{5}$ : The way of realization. The alphabet of all the parameters, except for the parameter $S_{2}$, is a bounded set of meanings, determined in case of the parameter $S_{1}$ from the nomenclature of the realized good $\{T\}$; for $S_{3}$-from the structure of the place of good realization $\{C\}$; for $S_{4}-$ from forecast period, determining the set $\{D\}$ at time scale. Parameter $S_{5}$ characterizes the way of good realization from the set $\{H\}$. Parameter $S_{2}$, shows the quantity of the realized good and not expressed by the set. 


\section{CONCLUSION}

The paper meets the challenge of developing a hybrid model of consumer satisfaction on the market. The model is based on the characteristics assessed statistically and by experts, indicators selected using fuzzy modeling, human needs system to ensure problem solving efficiency, namely, improve customer service quality, increase profits of the trading enterprise and ensure seamless customer and market interaction. When testing the model through 55 cycles of product promotion control with a total time of 4.5 years problem solving efficiency has been improved with ISSDM [15].

The environment modeling technique proposed differs from the analogous ones in combination of fuzzy and simulation modeling components and goods marketing optimization based on the integral efficiency criterion to exercise the fuzzy control of problem solving under great uncertainty. The structure of the simulation-optimization model with human needs system taken into account has been stated [16] to be a fundamental scientific research result and can be applied (if adjusted) in other areas to assess human needs.

This work is done by the support of SibSUTIS internal grant on project «Management in social and economic systems».

\section{REFERENCES}

1. Yarushkina, N. (2000) Soft Computing and Complex System Analysis// International Journal General Systems, 30, 1, 71-88.

2. Makin, I. (2011) Behavioural interpreting the compromise. Human and labour, 11, 68-71.

3. Crabbe, F.L. (2004) Optimal and non-optimal compromise strategies in action selection. In: Schaal S, et al., editors. From animals to animates 8: Proc. Eighth Int. Conf. on Simulation of Adaptive Behavior, 233-242.

4. Tshilidzi, Marwala (2013). Economic Modeling Using Artificial Intelligence Methods. Springer-Verlag: London, 263.

5. Yotaro, Hatamura \& Kenji, lino. (2006). Decision-Making in Engineering Design: Theory and Practice. Birkhuser, 265.

6. Zadeh, L.A., Fu, K.S., Tanaka, K. and Shimura, M. Fuzzy sets and their applications to Cognitive and Decision Processes. Academic Press: New York, 1975.

7. Alex, A. (2001). A Survey of Evolutionary Algorithms for Data Mining and Knowledge Discovery. Retrieved from http://citeseer.ist.psu.edu/freitas01survey.html.

8. Surmann H., Maniadakis M. (2001). Learning feed-forward and recurrent fuzzy systems: A genetic approach. Journal of System Architecture, 47, 649-662.

9. Omelchenko, A.A., Poletaykin, A.N. (2010). Genetic algorithm of making the optimal plan of computer technique realization. Questions of applied mathematics and mathematical modeling: collection of scientific papers. Dnepropetrovsk: Publishing office of Dnepropetrovsk National University, 221-231.

10. Gavrilova T.A., Khoroshevskiy V.F. (2001). Knowledge bases of intellectual systems. Saint-Petersburg: Piter, 384.

11. Jin Hyun Son, Myoung Ho Kim. (2004). An adaptable vertical partitioning method in distributed systems // Journal of Systems and Software. 73, 3, 551-561.

12. Surmann H. \& Maniadakis M. (2001) Learning feed-forward and recurrent fuzzy systems: A genetic approach. Journal of System Architecture, 47, 649-662.

13. Amosov, N.M. (1983). Human nature. Kiev: Naukova dumka, 223.

14. Maslow, A.H. (1997) Dominance, self-esteem, self-actualization: germinal papers. Monterey, CA.

15. Poletaikin, A.N. (2015). Hybrid approach to the building the system of the support of decision making by the goods promotion to the regional market. Vestnik SibSUTIS, 1, 45-59.

16. Poletaikin A.N., Nikiforova A.V. (2015). Decision making support system development in trade industry using hybrid models // Proceedings of XVI All-Russia conference for young scientists in mathematical modeling, Krasnoyarsk, Russia. 28-30 Oct. 2015. Novosibirsk: Institute of Computational Technologies SB RAS, 2015. - P. 86-87. 